\title{
A MODEL OF THE HOLOGRAPHIC PRINCIPLE: RANDOMNESS AND ADDITIONAL DIMENSION
}

\author{
ABRAHAM BOYARSKY, PAWEŁ GÓRA, AND HARALD PROPPE
}

\begin{abstract}
In recent years an idea has emerged that a system in a 3 dimensional space can be described from an information point of view by a system on its 2 dimensional boundary. This mysterious correspondence is called the Holographic Principle and has had profound effects in string theory and our perception of space-time. In this note we describe a purely mathematical model of the Holographic Principle using ideas from nonlinear dynamical systems theory. We show that a random map on the surface $S^{2}$ of a three dimensional open ball $\mathbf{B}$ has a natural counterpart in $\mathbf{B}$, and the two maps acting in different dimensional spaces have the same entropy. We can reverse this construction if we start with a special 3 dimensional map in $\mathbf{B}$ called a skew product. The key idea is to use the randomness, as imbedded in the parameter of the 2 dimensional random map, to define a third dimension. The main result shows that if we start with an arbitrary dynamical system in B with entropy E we can construct a random map on $S^{2}$ whose entropy is arbitrarily close to E.
\end{abstract}

\section{INTRODUCTION}

The Holographic Principle originated in the study of black holes through the work of Bekenstein and Hawking in the 1970s. It claims that all the information contained in some region of space can be represented as a "hologram" - a theory defined on the boundary of that region. Equivalence means that the information or entropic content of these two apparently different descriptions of nature is the same.

In this note we use the tools of nonlinear dynamical systems theory to consider a possible approach to a mathematical model of the Holographic Principle in a setting in which the underlying space is a (punctured) ball $\mathbf{B} \subset \mathbb{R}^{3}$ and its boundary sphere $S^{2}$. This choice is relatively simple but also appears to be reasonably natural (the missing centre of the ball can be thought of as corresponding to the singularity of a black hole).

In Section 2 we consider a 2 dimensional discrete time dynamical system on the boundary sphere $S^{2}$. We want to model fluctuations analogous to quantum effects near a black hole and we do this by starting with a deterministic map and adding random perturbations of this process, that is, by a random map [5], all of whose members are close to some central map. A random map is a family of maps selected randomly at each time iteration with probability $\nu$. Random maps can be used to model a wide range of dynamical behavior, from deterministic, to chaotic, to truly

Date: November 3, 2009.

Key words and phrases. holographic principle, random maps, skew products, entropy.

The research of the authors was supported by NSERC grants. 
random behavior. Indeed, any stationary discrete time process can be represented probabilistically by a deterministic chaotic map [4].

Our main construction shows that the random map on $S^{2}$ has a natural counterpart in the 3 dimensional system in $\mathbf{B}$, namely a skew product map. Although the random map on the surface of $\mathbf{B}$ and the skew product map in the interior act on different dimensional spaces they have the same measure-theoretic entropy. We describe the key construction which utilizes the randomness embedded in the parameter labeling the family of maps in the 2 dimensional random map to define an additional third dimension. This correspondence between randomness on $S^{2}$ and the emergence of a third dimension via the skew product construction is the main idea of our dynamical systems model of the Holographic Principle in this context. The key connection between the two dynamical systems is carried by the measure $\nu$ which describes probabilistically the family of maps that constitutes the random map and also defines the dynamics in $\mathbf{B}$.

We then show that if we start with an arbitrary 3 dimensional dynamical system in $\mathbf{B}$ with entropy $\mathbf{E}$ we can construct a skew product map in $\mathbf{B}$ and the associated random map on $S^{2}$ whose entropies are arbitrarily close to E. We consider a special dynamical system in $\mathbf{B}$ characterized by a snap back repeller [8]. Such a repeller has the property that orbits are attracted to it then pushed away if they get too close. Such behavior may serve as a model for gravitational singularities in a black hole where at some small distance from the singularity the attractive effect of gravity is reversed and becomes repulsive.

In Section 3 we present a detailed example. In Section 4 we conclude with a few additional remarks of a more speculative nature linking these results to physics and the Holographic Principle.

\section{Fundamental Constructions}

We consider the three dimensional ball $\mathbf{B}$, without the centre, represented as a product $(0,1] \times S^{2}$, where $\{1\} \times S^{2}$ will simply be referred to as $S^{2}$, the two dimensional unit sphere .

Now, we describe the random map construction motivated in part by the behaviour of subatomic particles near the event horizon of a black hole. Let $\left\{\tau_{c}\right.$, $c \in(0,1]\}$, be a family of maps where $\tau_{c}: S^{2} \rightarrow S^{2}$ and let $\mu$ be a common $\tau_{c}$-invariant measure equivalent to the Lebesgue measure on $S^{2}$ and where the parameter $c \in[0,1]$ is chosen randomly with probability $\nu$, equivalent to Lebesgue measure on $[0,1]$. In the usual formulation the parameter family is a finite set of maps but for our purposes the family can be considered to be a continuum as in [11]. We may think of this family as consisting of a central map with all the other maps representing minor perturbations of this central member, providing a model for quantum perturbations. The random map is the process in which on each step one of the maps $\left\{\tau_{c}\right\}$ is chosen at random (according to $\nu$ ) and applied to the current state of the system.

Under suitable conditions on the maps, the random map admits an invariant measure which is absolutely continuous with respect to Lebesgue measure on $S^{2}$. This measure provides a description of the long term statistical behavior of the dynamics on the boundary (which represents the horizon of a black hole).

Our objective now is to relate the dynamics on the horizon $S^{2}$ to a corresponding three dimensional motion inside $\mathbf{B}$. To that end we define a map $g:[0,1] \rightarrow[0,1]$ 
such that $g(0) \neq 0$ and $g(1)=1$ and preserves measure $\nu$. Note that $g$ is a chaotic map that preserves the same measure which defines the random map on the surface of $\mathbf{B}$. The pair $(g, \nu)$ conveys the nonlocal correlation between the horizon photons and their partners inside the sphere.

Our objective is to define a map $F$ such that the entropy of the dynamics inside $\mathbf{B}$ is the same as the entropy of the dynamics on $S^{2}$. Let us define $F: \mathbf{B} \rightarrow \mathbf{B}$ as follows:

1) For $0<c<1$ we define $F$ as a skew product [1]:

$$
F(c, z)=\left(g(c), \tau_{c}(z)\right) ;
$$

2) For $c=1, F$ is a random map

$$
F(1, z)=\left(1, \tau_{d}(z)\right),
$$

where $d \in[0,1]$ is chosen randomly according to the probability measure $\nu$.

The definition of $F$ implies that the projection of the interior dynamics onto this surface of $\mathbf{B}$ is statistically identical to the random map dynamics which acts there. But actually more is implied, namely, that the randomness of the dynamics on the horizon is equivalent to the additional dimension which we have for the interior dynamics. Alternatively, the third dimension of the dynamics in $\mathbf{B}$ is imbedded in the randomness of the dynamics on $S^{2}$. Our first objective is to prove that the entropy of the three dimension dynamical system inside $\mathbf{B}$ is the same as the entropy of the two dimensional system on $S^{2}$.

We consider the entropy of the skew product map in the interior of $\mathbf{B}$. For any finite measurable partition $\eta$ of $S^{2}$ we define partition

$$
\eta_{c}^{n}=\bigvee_{k=0}^{n-1} \tau_{c}^{-1} \tau_{g(c)}^{-1} \ldots \tau_{g^{k}(c)}^{-1}(\eta) .
$$

Let

$$
h_{g}(F, \eta)=\inf _{n \geq 1} \frac{1}{n} \int_{(0,1)} H\left(\eta_{c}^{n}\right) d \nu(c)
$$

and

$$
h_{g}(F)=\sup _{\eta} h(F, \eta)
$$

The entropy of $F$ is [1]

$$
h_{\text {interior }}(F)=h_{\nu}(g)+h_{g}(F) .
$$

To determine the entropy of the random map on $S^{2}$, let $\mathcal{C}=(0,1]^{\mathbb{N}}=\{\bar{c}=$ $\left.\left(c_{1}, c_{2}, \ldots\right): c_{i} \in(0,1]\right\}$ and let $\sigma$ be the left shift on $\mathcal{C}:$

$$
\sigma\left(\left(c_{1}, c_{2}, c_{3}, \ldots\right)\right)=\left(c_{2}, c_{3}, \ldots\right)
$$

The random map $F(1, z)$ can be understood as a skew product

$$
F(1,(\bar{c}, z))=\left(1,\left(\sigma(\bar{c}), \tau_{c_{1}}(z)\right)\right.
$$

To find the entropy we proceed as above. For any finite measurable partition $\eta$ of $S^{2}$ we define the partition

$$
\eta_{\bar{c}}^{n}=\bigvee_{k=0}^{n-1} \tau_{c_{1}}^{-1} \tau_{c_{2}}^{-1} \ldots \tau_{c_{k}}^{-1}(\eta)
$$


and let

$$
h_{\sigma}(F, \eta)=\inf _{n \geq 1} \frac{1}{n} \int_{(0,1)} H\left(\eta_{\bar{c}}^{n}\right) d \bar{\nu}(\bar{c})
$$

where $\bar{\nu}=\nu^{\mathbb{N}}$. We put

$$
h_{\sigma}(F)=\sup _{\eta} h(F, \eta)
$$

The entropy of $F$ is now defined [6] as:

$$
h_{\text {surface }}(F)=h_{\sigma}(F) \text {. }
$$

Using the Kifer-Liu generalization [6] of Pesin's formula, we obtain:

Theorem 1. For the maps $\tau_{c}=T_{N, c}$ defined below, we have

$$
h_{\sigma}(F) \simeq h_{g}(F),
$$

with the difference less than or equal to

$$
2\left(\ln \sup \left|f_{N, c, \varepsilon}^{\prime}\right|-\ln \inf \left|f_{N, c, \varepsilon}^{\prime}\right|\right) \text {. }
$$

Proof. To find the entropy $h_{g}(F)$ we consider the random map $F_{g}$ on the $S^{2}$, which for every $c \in(0,1]$ and $z \in S^{2}$ produces the trajectory $t_{(c, z)}^{(g)}=z \rightarrow \tau_{c}(z) \rightarrow$ $\tau_{g(c)}\left(\tau_{c}(z)\right) \rightarrow \ldots$ All maps $\tau_{c}$ preserve the same measure $\mu$ and $g$ preserves measure $\nu$ which are both absolutely continuous with respect to Lebesgue measure. By Theorem 3.1.3 of [6] the entropy of $F_{g}$ (which is equal to $h_{g}(F)$ ) can be calculated as

$$
\int_{(0,1]} \int_{S^{2}}\left(\lambda_{g}^{(1)}(c, z)+\lambda_{g}^{(2)}(c, z)\right) d \mu(z) d \nu(c),
$$

where $\lambda_{g}^{(i)}(c, z)$ are the Lyapunov exponent calculated on the trajectory $t_{(c, z)}^{(g)}=\left(z_{0}, z_{1}, z_{2}, \ldots\right)$. If $z_{j}=\left(z_{j}^{(1)}, z_{j}^{(2)}\right), j=0,1,2, \ldots$, then

$$
\lambda_{g}^{(i)}(c, z)=\lim _{n \rightarrow \infty} \frac{1}{n} \sum_{j=0}^{n-1} \ln \left|f_{N, c, \varepsilon}^{\prime}\left(z_{j}^{(i)}\right)\right|, \quad i=1,2,
$$

exists for $\nu \times \mu$ almost every trajectory $t_{(c, z)}$ and obviously

$$
\ln \inf \left|f_{N, c, \varepsilon}^{\prime}\right| \leq \lambda_{g}^{(i)}(c, z) \leq \ln \sup \left|f_{N, c, \varepsilon}^{\prime}\right|
$$

Quite similarly, the entropy $h_{\sigma}(F)$ can be calculated as

$$
\int_{(0,1]} \int_{S^{2}}\left(\lambda_{\sigma}^{(1)}(\bar{c}, z)+\lambda_{\sigma}^{(2)}(\bar{c}, z)\right) d \mu(z) d \bar{\nu}(\bar{c}),
$$

where $\lambda_{\sigma}^{(i)}(\bar{c}, z)$ is defined as above but using the trajectory

$$
t_{(\bar{c}, z)}^{(\sigma)}=z \rightarrow \tau_{(\bar{c})}(z) \rightarrow \tau_{\sigma(\bar{c})}\left(\tau_{(\bar{c})}(z)\right) \rightarrow \ldots .
$$

As before $\lambda_{\sigma}^{(i)}(\bar{c}, z), i=1,2$, satisfy the inequalities (2.1). This shows that

$$
\left|h_{\sigma}(F)-h_{g}(F)\right| \leq 2\left(\ln \sup \left|f_{N, c, \varepsilon}^{\prime}\right|-\ln \inf \left|f_{N, c, \varepsilon}^{\prime}\right|\right) .
$$




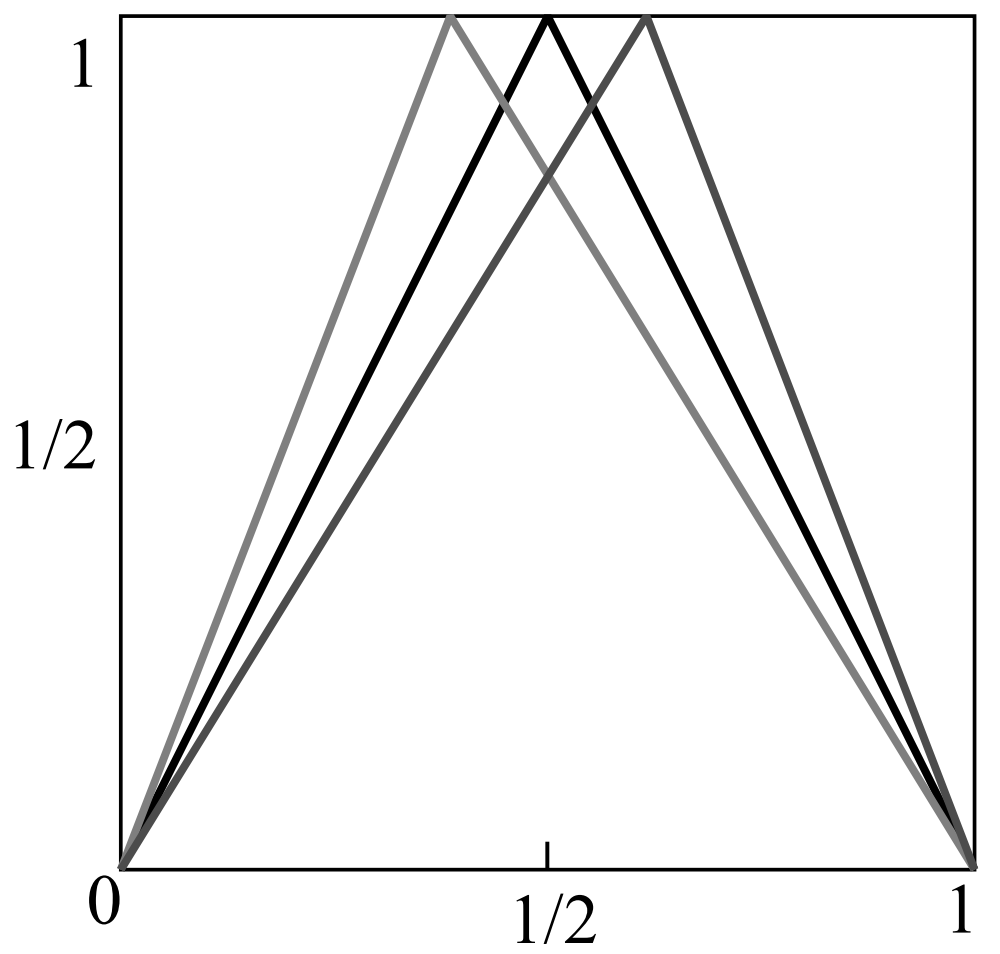

Figure 1. Maps $f_{c, \varepsilon}$ for $c=0,1 / 2,1$ and $\varepsilon=0.25$.

Using the above framework we now change our perspective. We consider a general 3 dimensional dynamical system inside $S^{3}$ with entropy $E$. Our objective is to construct a 2 dimensional system on $S^{2}$ which has the same entropy. Since the entropy $E$ is enormously large (of order $10^{66}$ ) it can be accurately approximated by a number of the form $2 \ln (N)$ for some even $N$. We consider the maps

$$
T_{c}(z)=f_{c, \varepsilon}(x) \times f_{c, \varepsilon}(y),
$$

on $S^{2}$ represented as the square $(0,1) \times(0,1)$, where

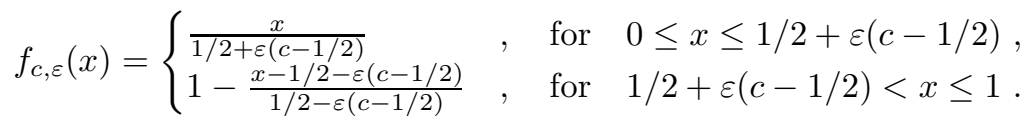

More generally, let us take an even number $N$ and define the map

$$
f_{N, c, \varepsilon}(x)=f_{c, \varepsilon}\left(\frac{N}{2} x-\operatorname{Int}\left(\frac{N}{2} x\right)\right), \quad x \in[0,1] .
$$

$f_{N, c, \varepsilon}$ preserves Lebesgue measure. Manipulating $\varepsilon$, we can make the entropy of $f_{N, c, \varepsilon}$ arbitrarily close to $\ln N$. Then, the map

$$
T_{N, c}=f_{N, c, \varepsilon} \times f_{N, c, \varepsilon},
$$

preserves Lebesgue measure on $[0,1] \times[0,1]$ and has entropy arbitrarily close to $2 \ln N$. 


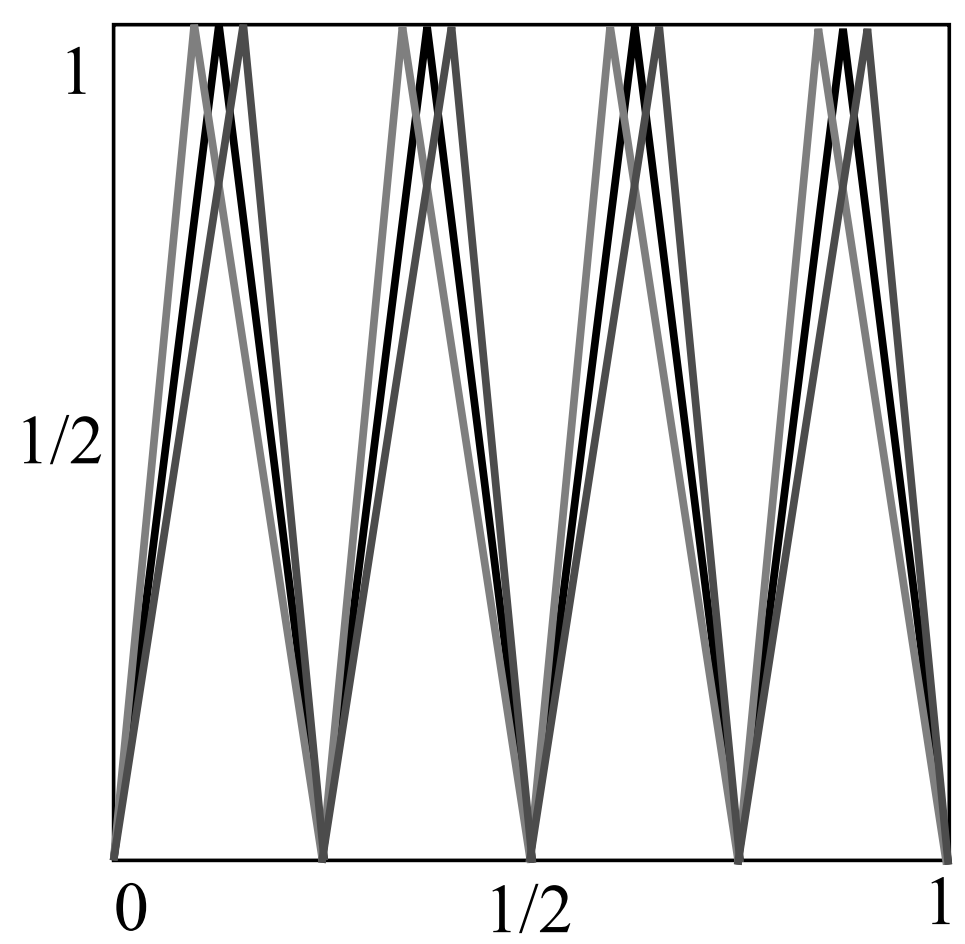

Figure 2. Maps $f_{8, c, \varepsilon}$ for $c=0,1 / 2,1$ and $\varepsilon=0.25$.

Although the gravitational singularity inside a black hole acts as a global attractor for the dynamics inside the horizon, it is also true that once the space at the singularity is packed too heavily with mass and energy it has a repulsive effect. This effect is well known and may be the cause of the big bang [10]. A way to represent this behavior is by the dynamical notion of a "snap-back" repeller [8], where almost every orbit approaches the point 0 and when it gets close enough it is repulsed from this point. We therefore define a map $g$ that captures this behavior. Let $g$ be defined as follows:

$$
g(c)= \begin{cases}1-\frac{(1-2 \delta) c}{\delta} & , \quad \text { for } 0 \leq c \leq \delta, \\ \frac{c-\delta}{1-\delta}, & \text { for } \delta<c \leq 1\end{cases}
$$

It can be shown that $g$ preserves a measure equivalent to the Lebesgue measure. Furthermore, careful choice of $g$ allows us to make the entropy $h_{\nu}(g)$ arbitrarily close to 0 . Then we have

$$
h_{\text {interior }}(F) \simeq h_{\text {surface }}(F),
$$

with error arbitrary close to $2\left(\ln \sup \left|f_{N, c, \varepsilon}^{\prime}\right|-\ln \inf \left|f_{N, c, \varepsilon}^{\prime}\right|\right)$, where $F$ in the interior of $\mathbf{B}$ is defined as a skew product

$$
F(c, z)=\left(g(c), \tau_{c}(z)\right),
$$

$\tau_{c}$ are maps of $S^{2}$ corresponding to maps $T_{N, c}=f_{N, c, \varepsilon} \times f_{N, c, \varepsilon}$ on $[0,1] \times[0,1]$ (see next section for the details). 


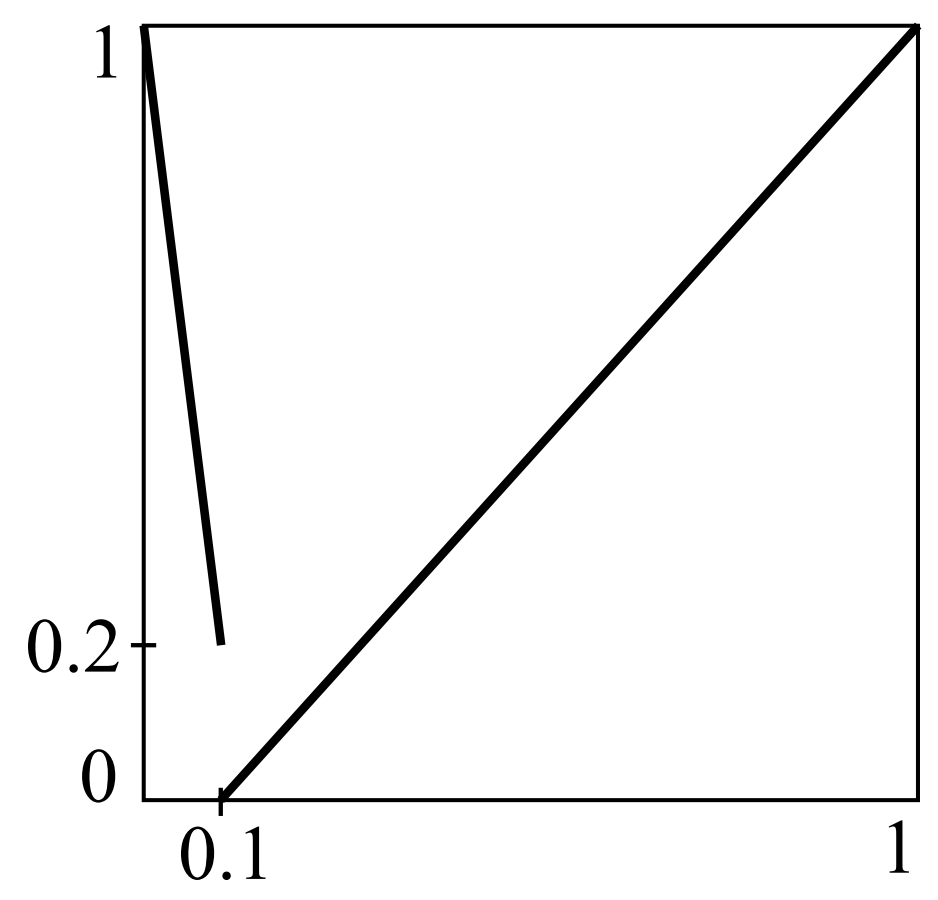

Figure 3. Map $g$ for $\delta=0.1$.

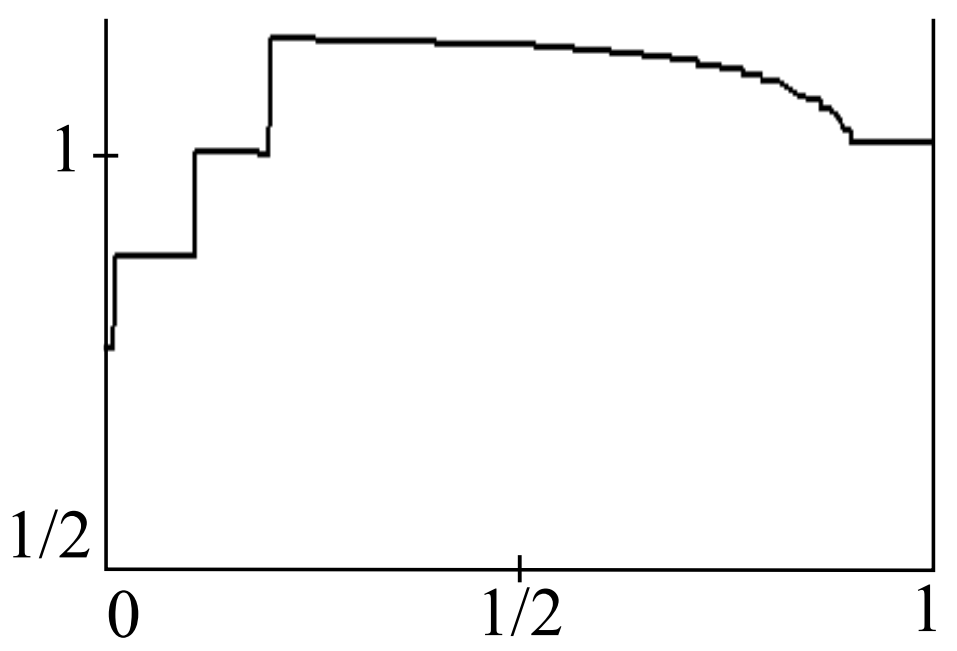

FiguRE 4. $g$-invariant density.

\section{Detailed Example}

We consider the Jabłoński maps $T_{c, \varepsilon}(z)$ of the previous section which can be written in a simpler form as $T_{\hat{c}}(z)$ with $\hat{c}=\varepsilon(c-1 / 2)+1 / 2$. We write $T_{c}(x, y)$ 
instead of $T_{\hat{c}}(z)$; i.e. $T_{c}(x, y)=\left(f_{c}(x), f_{c}(y)\right)$ on the unit square where

$$
f_{c}(x)=\left\{\begin{array}{cl}
\frac{x}{c} & 0 \leq x \leq c ; \quad c \in(0,1) \\
\frac{x-1}{c-1} & c \leq x \leq 1 ; \quad c \in(0,1)
\end{array}\right.
$$

(we may consider the $f_{c}$ to be perturbations of the standard tent map about $c_{0}=$ $1 / 2$ ) and note that if $R_{i}, i=1, \cdots, 4$ is one of the rectangles $J \times L$ where each of $J, L$ is either $[0, c]$ or $[c, 1]$ then $T_{c}$ maps $R_{i}$ homeomorphically onto $I \times I$. According to Theorem 3 of [2], Lebesgue measure is invariant under $T_{c}$. Moreover, if $\hat{T}_{c}$ is any other transformation mapping each of the $R_{i}$ homeomorphically onto $I$ and mapping $\{0,1\} \times I$ into $\{0\} \times I$ and $I \times\{0,1\}$ into $I \times\{0\}$ (and therefore mapping the boundary of the square into the boundary) then $T$ and $\hat{T}$ are topologically conjugate and Lebesgue measure is also invariant under $\hat{T}$.

Now let $k:(0,1) \rightarrow \mathbb{R}$ be any homeomorphism, e.g. $x \mapsto \tan (\pi(x-1 / 2))$, and extend to $I \rightarrow \mathbb{R} \cup\{\infty\}$. Define $K: I \times I \rightarrow \mathbb{R}^{2} \cup\{\infty\}$ by $K(x, y)=(k(x), k(y))$. Denote by $\sigma: S^{2} \rightarrow \mathbb{R}^{2} \cup\{\infty\}$ the stereographic projection from the unit sphere onto the extended plane.

$$
\sigma(X, Y, Z)=\left(\frac{X}{1-Z}, \frac{Y}{1-Z}\right) ; X^{2}+Y^{2}+Z^{2}=1,
$$

where the north pole $(0,0,1)$ corresponds to $\infty$. Then

$$
\sigma^{-1} \circ K: I \times I \rightarrow S^{2}
$$

is a homeomorphism on the interior of the square and maps the boundary of the square to $(0,0,1)$. We have the commutative diagram

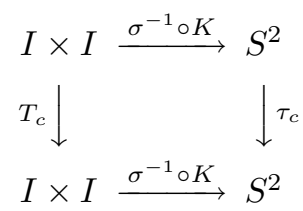

where each map $\tau_{c}$ is a 4 to 1 transformation on $S^{2} \backslash\{(0,0,1)\}$ with an absolutely continuous invariant measure induced by the Lebesgue measure on the unit square, and $(0,0,1)$ is a fixed point. If we use the specific example of $T_{c}$ and $K$ above it is possible to write an explicit expression for $\tau_{c}$ :

$$
\tau_{c}(X, Y, Z)= \begin{cases}\left(\frac{2 \alpha}{1+\alpha^{2}+\beta^{2}}, \frac{2 \beta}{1+\alpha^{2}+\beta^{2}}, \frac{-1+\alpha^{2}+\beta^{2}}{1+\alpha^{2}+\beta^{2}}\right) & Z \neq 1 \\ (0,0,1) & Z=1\end{cases}
$$

where

$$
\begin{aligned}
\alpha & =\tan \left(\pi f_{c}\left(\frac{1}{\pi} \tan ^{-1} \frac{X}{1-Z}+\frac{\pi}{2}\right)-\frac{\pi}{2}\right) \\
\beta & =\tan \left(\pi f_{c}\left(\frac{1}{\pi} \tan ^{-1} \frac{Y}{1-Z}+\frac{\pi}{2}\right)-\frac{\pi}{2}\right)
\end{aligned}
$$


We note that this also applies mutatis mutandis to the more general maps $T_{N, c}=$ $f_{N, c} \times f_{N, c}$ of the previous section (with $N^{2}$ rectangles; $N$ even), for which the entropy can be made arbitrarily close to $2 \ln N$.

\section{Concluding Remarks}

Do these results have any bearing on the Holographic Principle in physics? To be sure, the holographic paradigm is now being applied in a wide context, such as a superstring theory in an anti-de Sitter spacetime which is equivalent to a quantum field theory on its boundary. Malcadena [7] first conjectured such a relationship in 1997 and there are now similar examples of such correspondence in various dimensions. However, the Holographic Principle proposed by G. 't Hooft [14] and developed by Susskind [13] and others is based on the idea that the combination of quantum mechanics and gravity requires the three dimensional world to be an image of data that can be stored on a two dimensional projection much like a holographic image. It came about in a logical way as a resolution to several conundra, such as quantum decoherence, in quantum field theory when applied to fields in the black hole metric (Hawking radiation) [15].

This note could be a way to look at the Holographic Principle from a macroscopic statistical point of view, without considering the complex details of the underlying physics. Our setting can be interpreted as that of a black hole with a random map on the horizon $S^{2}$ representing the fluctuating quantum effects of Hawking radiation. For the dynamical system in the interior $\mathbf{B}$ we have made no attempt to model the (unknown and possibly unknowable) details of the dynamics inside a black hole, with one exception: the snap back repeller keeps the process away from the singularity at the centre; this seems to be a reasonable condition.

Our constructions arguably mimick longer-term quantum effects and lead to results that are intriguingly similar to what is claimed by the Holographic Principle: all the information (in the form of entropy) about the dynamics in the threedimensional space is captured in the two dimensional boundary. In particular, the main result states that if we start with an arbitrary dynamical system in $\mathbf{B}$ with entropy $E$ we can construct a random map on $S^{2}$ related in a natural way to the given dynamical system, with entropy that is mathematically arbitrarily close to $E$. But in the real world, once we are below the Planck scale (or whatever order of magnitude is needed), the two entropies become physically equal.

\section{REFERENCES}

[1] Abramov, L. M.; Rohlin, V. A.; Entropy of a skew product of mappings with invariant measure, (Russian. English summary) Vestnik Leningrad. Univ. 17 (1962), no. 7, 5-13, MR0140660 (25 \#4076).

[2] Boyarsky, A.; Byers, W.; Gauthier, P.; Higher-dimensional analogues of the tent maps, Nonlinear Anal. 11 (1987), no. 11, 1317-1324, MR0915528 (89h:58111).

[3] Boyarsky, Abraham; Góra, Paweł; Laws of chaos. Invariant measures and dynamical systems in one dimension, Probability and its Applications. Birkhäuser Boston, Inc., Boston, MA, 1997, MR1461536 (99a:58102).

[4] Boyarsky, Abraham ; Góra, Paweł; A description of stochastic systems using chaotic maps, J. Appl. Math. Stoch. Anal. 2004, no. 2, 137-141, MR2079262 (2006b:37066).

[5] Kifer, Yuri; Ergodic theory of random transformations, Progress in Probability and Statistics, 10. Birkhäuser Boston, Inc., Boston, MA, 1986, MR0884892 (89c:58069).

[6] Kifer, Yuri; Liu, Pei-Dong; Random dynamics. Handbook of dynamical systems, Vol. 1B, 379-499, Elsevier B. V., Amsterdam, 2006, MR2186245 (2008a:37002). 
[7] Maldacena, Juan; The Large-N Limit of Superconformal Field Theories and Supergravity, International Journal of Theoretical Physics 38, Number 4 (April, 1999), 1113-1133.

[8] Marotto, F. R; On redefining a snap-back repeller, Chaos Solitons Fractals 25 (2005), no. 1, 25-28, MR2123626 (2005k:37067).

[9] Mathur, S,D,. What exactly is the information Paradox?, arXiv:0803.2030V1 [hep-th] 13 Mar 2008.

[10] Bojowold, Martin; Follow the bouncing universe, Sci. Amer. 299 (October 2008), Number 4, 44-51.

[11] Morita, T; Random iteration of one-dimensional transformations, Osaka J. Math. 22 (1985), 481-518.

[12] Smolin, Lee, Three Roads to Quantum Mechanics, Basic Books, 2001.

[13] Susskind, L.; The World as a Hologram, J. Math. Physics 36 (1995), 63-77.

[14] 't Hooft, G.; Dimensional reduction in Quantum Gravity, arXiv:gr-qc/9310026v2 (1993).

[15] 't Hooft, G.; The Holographic Principle, arXiv:hep-th/0003004 (2000).

Department of Mathematics and Statistics, Concordia University, 1455 de Maisonneuve Blvd. West, Montreal, Quebec H3G 1M8, Canada

E-mail address: boyar@alcor.concordia.ca, pgora@mathstat.concordia.ca, proppe@alcor.concordia.ca 